\title{
Novel Versatile Smart Phone Based Microplate Readers for On-site Diagnoses
}

Qiangqiang $\mathrm{Fu}^{\mathrm{a}}$, Ze $\mathrm{Wu}^{\mathrm{a}}$, Xiuqing $\mathrm{Li}^{\mathrm{a}}$, Cuize $\mathrm{Yao}^{\mathrm{a}}$, Shiting $\mathrm{Yu}^{\mathrm{a}}$, Wei Xiao ${ }^{\mathrm{a}}$, Yong Tang ${ }^{\mathrm{a}, \mathrm{b}_{*}}$

${ }^{a}$ Department of Bioengineering, Guangdong Province Key Laboratory of Molecular Immunology and Antibody Engineering, Jinan University, Guangzhou 510632, PR China.

${ }^{b}$ Institute of Biotranslational Medicine, Jinan University, Guangzhou, 510632, PR China

Corresponding authors: Fax: (+86)-20-85227003; Tel: (+86)-20-85227003; E-mail: tyjaq7926@163.com.

Abstract: Microplate readers are important diagnostic instruments, used intensively for various readout test kits (biochemical analysis kits and ELISA kits). However, due to their expensive and non-portability, commercial microplate readers are unavailable for home testing, community and rural hospitals, especially in developing countries. In this study, to provide a field-portable, costeffective and versatile diagnostic tool, we reported a novel smart phone based microplate reader. The basic principle of this devise relies on a smart phone's optical sensor that measures transmitted light intensities of liquid samples. To prove the validity of these devises, developed smart phone based microplate readers were applied to readout results of various analytical targets. These targets included analanine aminotransferase (ALT; limit of detection (LOD) was 17.54 U/L), alkaline phosphatase (AKP; LOD was $15.56 \mathrm{U} / \mathrm{L}$ ), creatinine (LOD was $1.35 \mu \mathrm{M}$ ), bovine serum albumin (BSA; LOD was $0.0041 \mathrm{mg} / \mathrm{mL}$ ), prostate specific antigen (PSA; LOD was $0.76 \mathrm{pg} / \mathrm{mL}$ ), and ractopamine (Rac; LOD was $0.31 \mathrm{ng} / \mathrm{mL}$ ). The developed smart phone based microplate readers are versatile, portable, and inexpensive; they are unique because of their ability to perform under circumstances where resources and expertise are limited.

Key words: Smart phone, microplate reader, optical sensor, biochemical analysis kits, ELISA kits

\section{Introduction}

In modern society, human beings have faced enormous health challenges, mainly due to increasing incidences of cancer, cardiovascular diseases, obesity, autoimmune diseases, and infectious diseases (HIV, HBV, Ebola), etc.(Drain et al. 2014; McNerney and Daley 2011; Peeling and Mabey 2010). In the past few decades, various technologies and tools have been reported and improved for diagnoses (Fu et al. 2015; Jia et al. 2009; Liang et al. 2015; Tian et al. 2016). The microplate reader is still one of the most common instruments applied to clinical diagnoses, 
because of its accuracy and precision. Results of various test kits, including biochemical analysis kits and ELISA kits that related to hundreds of important analytes (e.g., metabolites, proteins, enzymes) were readout by microplate readers. However, current commercial microplate readers are unavailable for testing in homes, some community and rural hospitals, especially in developing countries. This is probably because commercial microplate readers are expensive, non-portable, and require high maintenance. Therefore, a simple, cost effective, easy-to-use, and portable microplate reader would be a welcomed diagnostic tool for providing field medical services.

Smart phone based biosensors would be cost effective, portable diagnostic tools, especially when used in developing countries and locations with limited resources (Bastawrous and Armstrong 2013; Coskun and Ozcan 2014; Gopinath et al. 2014; Ma et al. 2016; Preechaburana et al. 2014; Vashist et al. 2014). Smart phones equipped with sophisticated permanent physical sensors, including magnetometers, accelerometers, optical sensors, and gyroscopes, together with Global Positioning Systems, would make smart phones versatile biosensor platforms. Currently, smart phones play important roles for light microscopy (Walzik et al. 2015; Zhu et al. 2013), single-molecule microscopy (Vashist et al. 2015; Wei et al. 2014), cell imaging (Walzik et al. 2015), cytometry (Zhu et al. 2011), bacteria detection (Coskun et al. 2013; Rajendran et al. 2014), colorimetric detection (Berg et al. 2015; Yang et al. 2016), ELISA kits (Berg et al. 2015; Christodouleas et al. 2015), lateral flow devices (Yu et al. 2015; Zangheri et al. 2015), genetic analysis (Shu et al. 2015) and microfluidic chips (Barbosa et al. 2015; Song et al. 2014). In addition, smart phones can wirelessly integrate the Internet, establishing the smart phone as a ubiquitous platform for developing various simple, low-cost, easy-to-use, and portable instruments for clinical diagnoses. In previously reported smart phone based biosensors, results of assay kits obtained by digital cameras were analyzed by a smart phone application. Although many calibration methods, such as dark-hood, mapping algorithm, and paralleled analysis were used, the above described methods had difficulty obtaining explicit quantifications, due to the smart phone's imaging capabilities, which couldn't be performed precisely until recently (Zhang 
and Liu 2016).

Therefore, to provide an accurate smart phone based microplate reader, we used an optical sensor, normally installed in smart phones, to measure transmitted light intensities of liquid assay samples (figure 1a). Current optical sensors of smart phone are positioned on the top of screens and can sense light from $340 \mathrm{~nm}$ to $680 \mathrm{~nm}$. The cylindrical shape of the developed smart phone based microplate reader has the following dimensions: diameter $14 \mathrm{~mm}$; length $38 \mathrm{~mm}$; weight $3.5 \mathrm{~g}$. To prove the validity of these devises, developed smart phone based microplate readers were applied to readout results of various analytical targets; these included analanine aminotransferase (ALT), alkaline phosphatase (AKP), creatinine, bovine serum albumin (BSA), prostate specific antigen (PSA), and ractopamine (Rac). Selected assays for these targets were well established and used intensively for practical diagnoses. These molecules were important health indicators, demonstrating that developed smart phone based microplate readers could be used widely in home testing and personal healthcare.

\section{Materials and Methods}

\subsection{Materials}

BCA assay kit, creatinine assay kit, ALT assay kit, and APK assay kit were purchased from Nanjing Jiancheng Biotech. The commercial microplates were purchased from JET BIOFIL. Antihuman PSA monoclonal antibodies (mAbs) and PSA were obtained from Shanghai Lingchao Biological Technology Co., LTD. Anti-Rac monoclonal antibodies (mAbs) and coating antigen were prepared in our lab. Serum samples were collected from the Department of Laboratory Medicine of Guangzhou Overseas Chinese Hospital. LEDs (450 nm, $510 \mathrm{~nm}, 560 \mathrm{~nm}$ ) were purchased from Shenzhen OCtai Co., LTD. A battery ( 3 V) was purchased from VSAI. Black plastic rods with diameters of $12 \mathrm{~mm}$, transparent white plastic pipes with diameters of $14 \mathrm{~mm}$, black plastic pipes with diameters of $12 \mathrm{~mm}$, resistor, switch, and wires were purchased from a local store. The absorbance of liquid assays was analyzed by using a Synergy H1 Hybrid Multi-mode Microplate Reader (Bio-Tek Instruments, Inc.). In our design, we chose the HUAWEI Honor 6 as the starting based smart phone for fabricating the smart phone based microplate reader. 


\subsection{Design of the smart phone based microplate reader}

When designing the smart phone based microplate reader for BCA assay kit, and ELISA, LEDs' emission lights were $560 \mathrm{~nm}$ and $450 \mathrm{~nm}$, and resistances of used resistors were $10000 \Omega$ and 50 $000 \Omega$, respectively. When designing the smart phone based microplate reader for creatinine assay kit, AKP assay kit, and ALT assay kit, LEDs' emission lights were $510 \mathrm{~nm}$, and resistances of used resistors were $10000 \Omega$.

\subsection{Performance of smart phone based microplate readers}

Procedures for detecting BSA and creatinine in urine, and AKP and ALT in serum are referred to in their instructions. Procedures of ELISA kits for detecting PSA and Rac were referred in previous research (Jia et al. 2009). Details of these experiments are explained in Supplementary Data. In our study, considering the requirement of home testing, community and rural hospitals, we fabricated a portable temperature control device $(70 \mathrm{~mm} * 70 \mathrm{~mm} * 80 \mathrm{~mm})$ for incubating the liquid samples.

\section{Results and Discussion}

\subsection{The basic work principle of the smart phone based microplate reader}




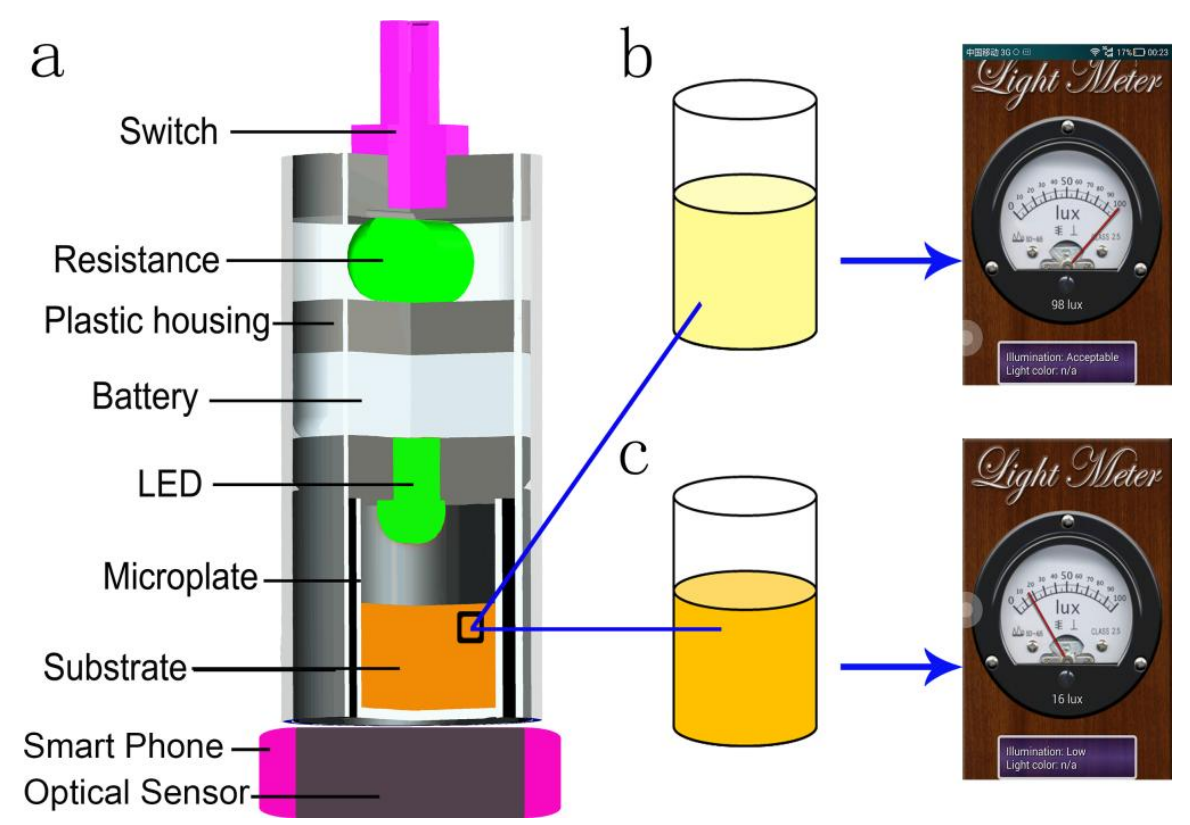

Figure1. Scheme of smart phone based microplate reader: a. Internal structure of the attachment; b. Measured transmitted light intensity was high when absorbance of analyzed assay was low; c. Measured transmitted light intensity was low when absorbance of analyzed assay was high.

The principle of the smart phone based microplate reader is displayed in figure 1 . When the assay kit was completed, the liquid assay in the microplate was put into the smart phone based microplate reader. Then the microplate reader was attached to the optical sensor of the smart phone, which formed an analytical system. Once the microplate reader's switch was turned on, intensities of transmitted light were measured by the smart phone's optical sensor. In this study, the optical sensor of the smart phone was located in the upper left of the screen (figure 1a, figure 2 a). When the liquid assay absorbance was low, measured transmitted light intensity was high (figure 1b), and when liquid assay absorbance was high, measured transmitted light intensity was low (figure 1c). Emission wavelengths of selected LEDs in smart phone based microplate readers were maximum absorption wavelengths of liquid assays. In this study, for ALT, AKP and creatinine assays, maximum absorption wavelengths of liquid samples were $510 \mathrm{~nm}$. Therefore, selected emission wavelengths of LEDs in the smart phone based microplate reader were $510 \mathrm{~nm}$. In addition, emission wavelengths of LEDs in smart phone based microplate readers for BCA assay kit and ELISA kits were $560 \mathrm{~nm}$ and $450 \mathrm{~nm}$, respectively. In the smart 
phone based microplate reader, a resistor was used to adjust light intensity and a button cell $(3 \mathrm{~V})$ was used to power LEDs (figure 1a). All of these electrical and optical components were assembled in a cylindrical plastic housing (diameter was $14 \mathrm{~mm}$ and length was $38 \mathrm{~mm}$ ) (figure 1a; figure 2a), with a weight of $3.5 \mathrm{~g}$. A free android application, Light Meter, was applied to display light intensity on the phone's screen.
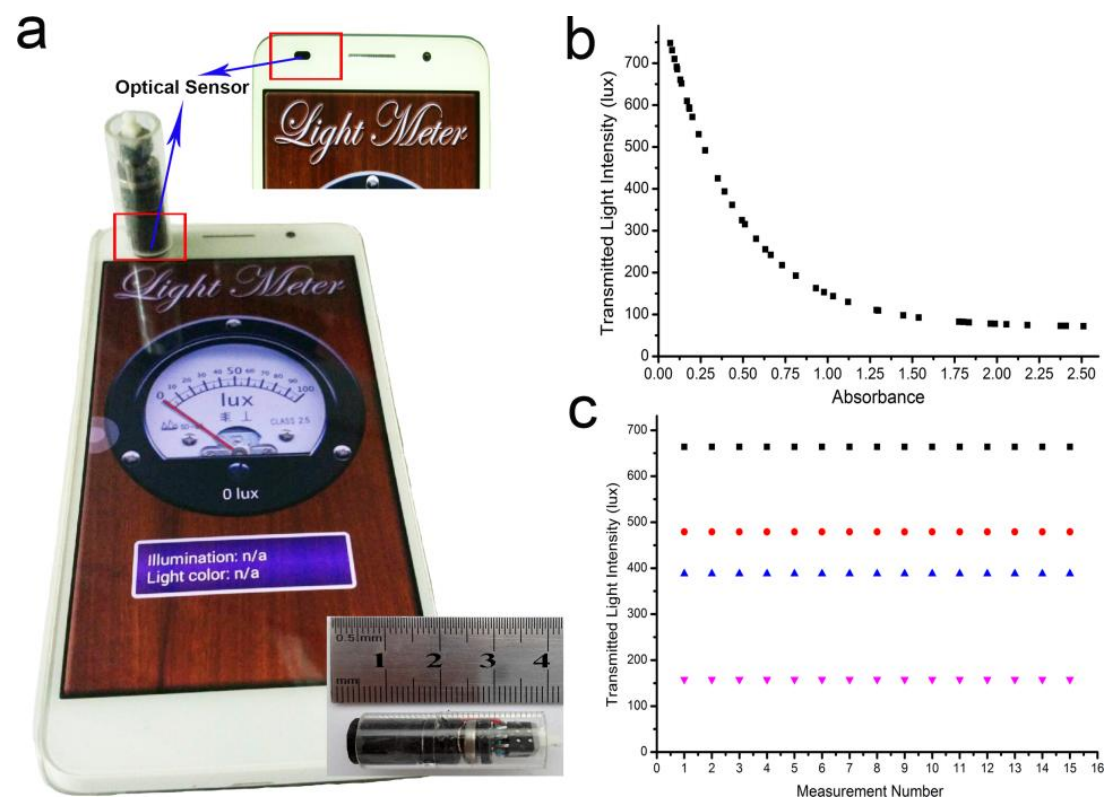

Figure 2. a. Photograph of the actual microplate reader run on an Android-based smart phone. The screen of the smart phone displays a typical image of the sample. The optical sensor in this study was located in the upper left of the phone's screen; b. Corresponding relationship of measured results from smart phone based microplate reader and commercial microplate reader. As measured absorbance of commercial microplate reader increased, intensities of transmitted light measured by the smart phone based microplate reader index decreased; $d$. Stability of smart phone based microplate reader.

In order to study the accuracy of the smart phone based microplate reader, AKP was analyzed by assay kits; their results were readout by the smart phone based microplate reader and commercial microplate reader, respectively. As measured absorbance increased, transmitted light intensity measured by the smart phone based microplate reader decreased (figure $2 \mathrm{~b}$ ). The resolution of the smart phone based microplate reader is defined as the ratio of measured absorbance change to measured transmitted light intensity changes. When absorbance values of AKP assay substrate increased, corresponding resolutions of the smart 
phone based microplate reader decreased. Resolution of the used commercial microplate reader was 0.001 . When absorbance liquid assays were higher than 1.4 , resolutions of the commercial microplate reader were higher than those of the smart phone based microplate reader. This was because the resolution of the used android application was low (1 lux). If a high resolution Android application had been used, the smart phone based microplate reader would have produced more accurate results. Transmitted light intensities of AKP assays were measured with 10 duplicates and results of each measurement were equal (figure $2 \mathrm{c}$ ), thus, proving the smart phone based microplate reader's stability.

\subsection{Performance of smart phone based microplate reader for detection of ALT and AKP}

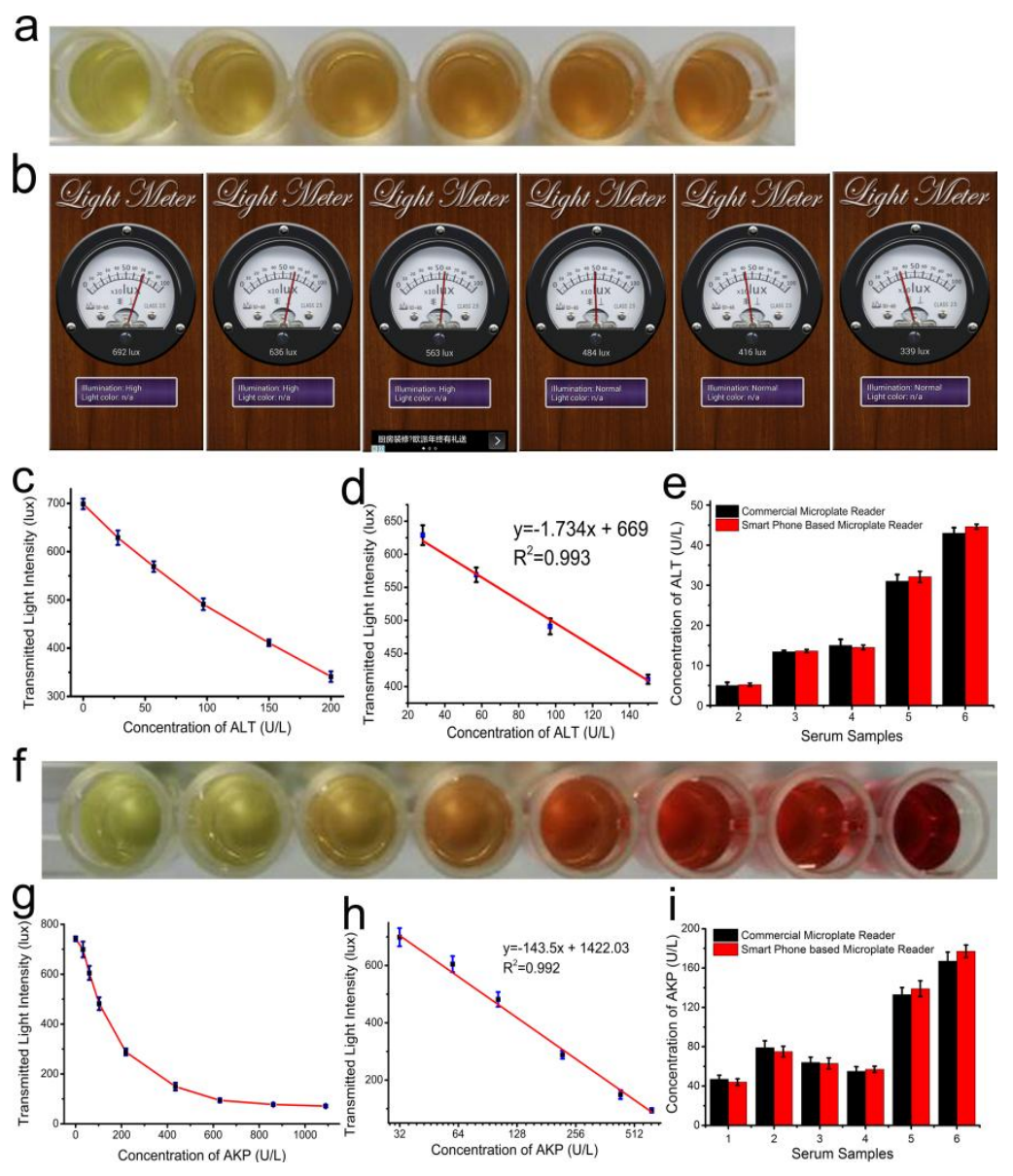

Figure 3. Performance of smart phone based microplate reader for detection of ALT and AKP; a. Photograph of assays for detecting different concentrations of ALT; b. Smart phone screenshots of assays for detecting different concentrations of ALT. From left to right, transmitted light intensity is 692 lux, 636 lux, 563 lux, 484 lux, 416 lux and 339 lux, respectively; c. Measured transmitted light intensities of smart phone based microplate reader for detecting different concentrations of ALT; $d$. Calibration curve of ALT assay readout by smart phone based 
microplate reader. Each value represents the mean of three independent experiments $(n=3)$; e. Comparison of smart phone based microplate reader and commercial microplate reader for readout ALT assays in serum samples; f. Photograph of assays for detecting different concentrations of APK; g. Transmitted light intensities measured by smart phone based microplate reader for different concentrations of AKP; h. Calibration curve of AKP assay readout by smart phone based microplate reader. Each value represents the mean of three independent experiments $(n=3)$; i. Comparison of smart phone based microplate reader and commercial microplate reader for detecting AKP in serum samples.

Concentrations of enzymes are important health indicators for a series of diseases. Colorimetric biochemical assay kits provide a quick and sensitive method for measuring enzymes in various biological samples. When there are damaged cells, such as in acute hepatitis, chronic hepatitis, obstructive jaundice, carcinoma of the liver, and myocardial infarction, ALT may leak into the blood and its levels will become significantly elevated. Therefore, ALT was often used to diagnose liver injuries and long-term liver diseases (Chadban et al. 2003; Prati et al. 2002; Sorbi et al. 1999). In addition, abnormal levels of AKP in blood often indicate liver and/or bone problems (rickets, osteomalacia, bone cancer, bone cancer metastasis, liver cancer, cirrhosis of the liver, andbile canaliculi) (Fishman et al. 1965; Kaplan 1972;Pekarthy et al. 1972). In our research, results of ALT assays and AKP assays were obtained by using a smart phone based microplate reader (figure 3a, figure 3e). As shown in figure $3 \mathrm{~b}-\mathrm{c}$ and figure $3 \mathrm{~g}$, along with increasing enzyme concentrations, intensities of measured transmitted light decreased. Transmitted light intensities were applied to quantitatively measured concentrations of ALT and AKP. For detecting ALT, a good linear correlation $\left(R^{2}=0.993\right)$ between the transmitted light intensities and ALT concentrations was obtained within $28 \mathrm{U} / \mathrm{L}-150 \mathrm{U} / \mathrm{L}$ (figure 3d). The calculated limit of detection (LOD) of the ALT assays was $17.54 \mathrm{U} / \mathrm{L}$. For detecting $A K P$, a good linear correlation $\left(R^{2}=0.992\right)$ between the transmitted light intensities and AKP concentration was obtained within $32 \mathrm{U} / \mathrm{L}-625 \mathrm{U} / \mathrm{L}$ (figure 3h). The LOD of the AKP assays was $15.56 \mathrm{U} / \mathrm{L}$. Concentrations of ALT and AKP in serum samples were analyzed by corresponding assay kits, and their results were readout by the smart phone based microplate reader and commercial microplate reader, respectively (figure $3 e$ and figure 3i). Results from these two devises showed an excellent correlation, indicating that the smart phone 
based microplate readers could detect ALT and AKP in clinical diagnoses.

\subsection{Performance of smart phone based microplate reader for readout BCA assay and creatinine}

assay.

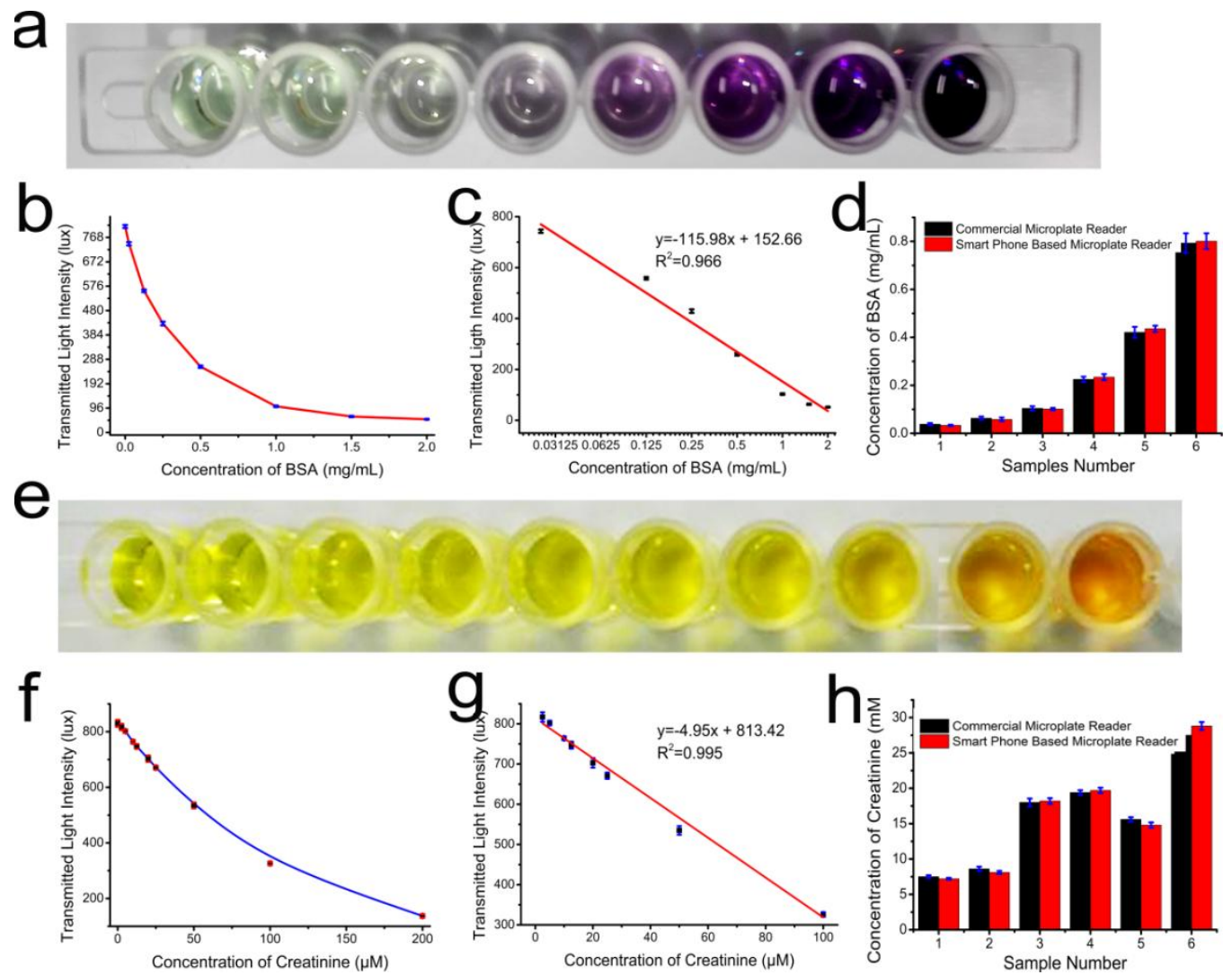

Figure 4. Performance of smart phone based microplate reader for readout BCA assay and creatinine assay; a. Photograph of BCA assay for different concentrations of BSA; $b$. Transmitted light measured by the smart phone based microplate reader for detection of different concentrations of BSA; c. Calibration curve of BCA assay for detecting BSA readout by smart phone based microplate reader. Each value represents the mean of three independent experiments $(n=3)$; d. Comparison of BCA assay's results readout by smart phone based microplate reader and commercial microplate readerin urine; e. Photograph of assays for different concentrations of creatinine; f. Transmitted light measuredby smart phone based microplate reader for detection of different concentrations of creatinine; g. Calibration curve of creatinine assay readout by the smart phone based microplate reader. Each value represents the mean of three independent experiments $(n=3) ; \mathrm{h}$. Comparison of results for detecting creatinine in urine readout by the smart phone based microplate reader and commercial microplate reader.

Abicinchoninic acid (BCA) assay kit is a commonly used method for protein detection. Concentrations of urinary protein are important indicators for many diseases (Chadban et al. 
2003; Schaub et al. 2004). In our study, we used the BCA kit to detect BSA and its results were readout by the smart phone based microplate reader (figure 4a-b). The linear detection range of the BCA assay for detecting BSA was from $0.02 \mathrm{mg} / \mathrm{mL}-1 \mathrm{mg} / \mathrm{mL}$ (figure 4c) and the LOD was $0.0041 \mathrm{mg} / \mathrm{mL}$. Different concentrations of BSA were spiked in urine samples and then determined by a BCA assay kit. Their results were readout by a smart phone based microplate reader and commercial microplate reader simultaneously. Results from these two instruments showed an excellent correlation (figure 4d), indicating that the smart phone based microplate reader was an effective tool for protein detection in urine. Creatinine concentrations in urine are important indicators for renal diseases (Donmez et al. 2015). In this research, we detected creatinine by using picric acid methods and results were readout by the smart phone based microplate reader (figure 4e). The linear detection range of the creatinine assay readout by the smart phone based microplate reader was from $2.5 \mu \mathrm{M}-100 \mu \mathrm{M}$ (figure $4 \mathrm{~g}$ ) and its LOD was 1.35 $\mu \mathrm{M}$. Creatinine was analyzed in urine samples and readout, followed by the smart phone based microplate reader and commercial microplate reader, respectively. Measured concentrations of creatinine in urine from above two devises showed an excellent correlation (figure 4h), indicating the smart phone based microplate reader was an effective tool for detection of creatinine in urine samples.

\subsection{Performance of smart phone based microplate reader to readout ELISA kits for detection of PSA and Rac}




\section{a}
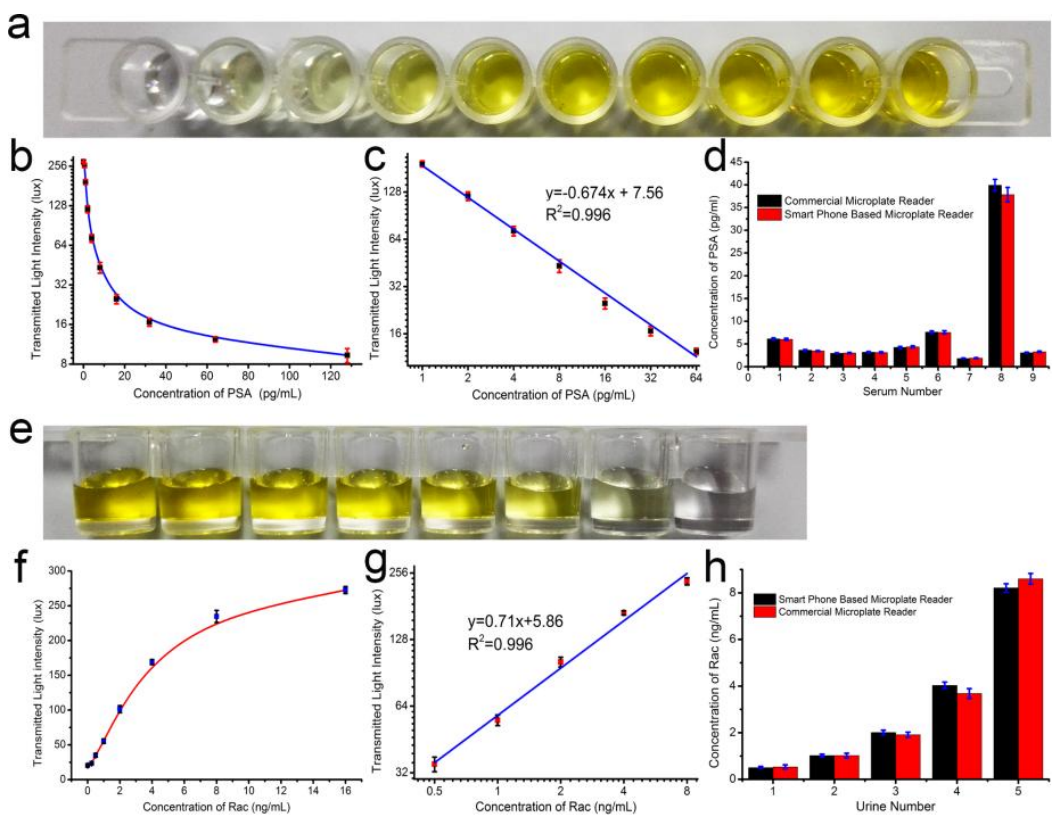

Figure 5. Performance of smart phone based microplate readers to readout ELISA kits; a. Photograph of ELISA for detecting different concentrations of PSA; $b$. Measured transmitted light intensities of smart phone based microplate reader for detecting different concentrations of PSA; c. Calibration curve of ELISA for detecting PSA readout by smart phone based microplate reader. Each value represents the mean of three independent experiments $(n=3)$; d. Comparison of PSA ELISA kit readouts by smart phone based microplate reader and commercial microplate reader; e. Photograph of ELISA for detecting different concentrations of Rac; f. Measured transmitted light intensities of smart phone based microplate reader for detecting different concentrations of Rac; g. Calibration curve of ELISA for detecting Rac readout by smart phone based microplate reader. Each value represents the mean of three independent experiments $(n=3) ; h$. Comparison of results of Rac ELISA kit readouts by smart phone based microplate reader and commercial microplate reader.

Enzyme-linked immunosorbent assay (ELISA) is one of the most important analytical techniques for clinical diagnoses, environmental monitoring, and food safety testing, due to its low-cost, high specificity, and high sensitivity. For detecting proteins, ELISA format is sandwich immunoassay, and for detecting small molecules, ELISA format is competitive immunoassay. In this study, to confirm that our developed smart phone based microplate reader could readout ELISA for protein detection, we prepared an ELISA kit to detect PSA (figure 5a). As shown in figure 5b, along with increasing PSA concentrations, intensities of transmitted light decreased. Measured transmitted light intensities were employed to quantitative analysis concentrations of PSA. Results showed that linear detection range of the ELISA kit for PSA was from $1 \mathrm{pg} / \mathrm{mL}-64$ 
$\mathrm{pg} / \mathrm{mL}$ (figure $5 \mathrm{c}$ ) and the LOD was $0.76 \mathrm{pg} / \mathrm{mL}$. Specificity of ELISA for detecting PSA was studied and results showed a high selectivity (figure S13). In order to confirm that our developed devise could readout ELISA for competitive immunoassays, concentrations of Rac were detected by an ELISA kit (figure 5 e). The linear detection range of ELISA for Rac was from $0.5 \mathrm{ng} / \mathrm{mL}-8 \mathrm{ng} / \mathrm{mL}$ (figure $5 \mathrm{f}$ ) and LOD was $0.31 \mathrm{ng} / \mathrm{mL}$. Specificity of ELISA for detecting Rac was studied and results showed a high selectivity (figure S14). PSA in serum samples and Rac in urine samples were measured by ELISAs and results were readout by the smart phone based microplate reader and commercial microplate reader simultaneously. Results from these two instruments showed excellent correlation (figure $5 \mathrm{~d}$ and $\mathrm{h}$ ), indicating the smart phone based microplate reader could readout ELISA kits in clinical samples.

\subsection{Comparison of commercial microplate reader and smart phone based microplate reader}

Table 1. Comparison of results from the commercial microplate reader and smart phone based microplate reader

\begin{tabular}{|c|c|c|c|c|c|}
\hline Analyte & Apparatus & LDR & $\mathrm{R}^{2}$ & LOD & Normal level \\
\hline \multirow{2}{*}{ ALT } & $\mathrm{a}$ & $28 \mathrm{U} / \mathrm{L}-150 \mathrm{U} / \mathrm{L}$ & 0.993 & $17.54 \mathrm{U} / \mathrm{L}$ & \multirow{2}{*}{$0-40 \mathrm{U} / \mathrm{L}$} \\
\hline & $b$ & $28 \mathrm{U} / \mathrm{L}-150 \mathrm{U} / \mathrm{L}$ & 0.994 & $9.87 \mathrm{U} / \mathrm{L}$ & \\
\hline \multirow{2}{*}{ AKP } & a & $32 \mathrm{U} / \mathrm{L}-625 \mathrm{U} / \mathrm{L}$ & 0.992 & $15.56 \mathrm{U} / \mathrm{L}$ & \multirow{2}{*}{$40-200 \mathrm{U} / \mathrm{L}$} \\
\hline & $b$ & $32 \mathrm{U} / \mathrm{L}-1250 \mathrm{U} / \mathrm{L}$ & 0.999 & $13.8 \mathrm{U} / \mathrm{L}$ & \\
\hline \multirow{2}{*}{ BSA } & a & $0.02 \mathrm{mg} / \mathrm{mL}-1 \mathrm{mg} / \mathrm{mL}$ & 0.966 & $0.0041 \mathrm{mg} / \mathrm{mL}$ & \multirow{2}{*}{ less than $0.2 \mathrm{mg} / \mathrm{mL}$} \\
\hline & $b$ & $0.02 \mathrm{mg} / \mathrm{mL}-2 \mathrm{mg} / \mathrm{mL}$ & 0.999 & $0.0042 \mathrm{mg} / \mathrm{mL}$ & \\
\hline \multirow{2}{*}{ Creatinine } & a & $2.5 \mu \mathrm{M}-100 \mu \mathrm{M}$ & 0.995 & $1.35 \mu \mathrm{M}$ & \multirow{2}{*}{$5.3-18 \mathrm{mM}$} \\
\hline & $b$ & $2.5 \mu \mathrm{M}-200 \mu \mathrm{M}$ & 0.999 & $1.5 \mu \mathrm{M}$ & \\
\hline \multirow{2}{*}{ PSA } & a & $1 \mathrm{pg} / \mathrm{mL}-64 \mathrm{pg} / \mathrm{mL}$ & 0.996 & $0.76 \mathrm{pg} / \mathrm{mL}$ & \multirow{2}{*}{ less than $4 \mathrm{ng} / \mathrm{mL}$} \\
\hline & $b$ & $1 \mathrm{pg} / \mathrm{mL}-128 \mathrm{pg} / \mathrm{mL}$ & 0.998 & $0.59 \mathrm{pg} / \mathrm{mL}$ & \\
\hline \multirow{2}{*}{ Rac } & a & $0.5 \mathrm{ng} / \mathrm{mL}-8 \mathrm{ng} / \mathrm{mL}$ & 0.996 & $0.31 \mathrm{ng} / \mathrm{mL}$ & \multirow{2}{*}{ not detected } \\
\hline & $b$ & $0.5 \mathrm{ng} / \mathrm{mL}-8 \mathrm{ng} / \mathrm{mL}$ & 0.994 & $0.48 \mathrm{ng} / \mathrm{mL}$ & \\
\hline
\end{tabular}

Notes: Results are from three independent experiments $(n=3)$. Apparatus a: smart phone based microplate reader; apparatus b: commercial microplate reader; LDR: linear detection range; $\mathrm{R}^{2}$ : correlation coefficient; LOD: limit of detection. Details of results from commercial microplate reader are presented in the Supplementary Data.

Results from these two instruments are displayed in Table 1. Correlation coefficients from smart phone based microplate reader were lower than from commercial microplate reader. This is because of low resolution of used Android application (1 lux), which prevented it distinguishing slight changes in light intensity. Therefore, a higher resolution smart phone application is essential for future studies. In addition, the created smart phone application could store a 
calibration curve to convert measured transmitted light intensity into analyte concentrations. Time consumptions were essential for diagnostic techniques and were divided into two parts: assay times and measuring times. Assay times for these two instruments were equal. For measuring times, the smart phone based microplate reader needed about $3 \mathrm{~s}$ to readout 1 sample; whereas, the commercial microplate reader could readout 96 samples within $10 \mathrm{~s}$. When considered as a personal healthcare or home testing device, generally only a few samples needed to be detected; the overall time consumption of the smart phone based microplate reader was close to that of the commercial microplate reader.

\section{Conclusions}

In this study, we researched a spectrophotometric smart phone based microplate reader for point of care testing applications. Developed smart phone based microplate readers were used to readout the results of biochemical analysis kits (total proteins in urine, creatinine, ALT and AKP in serum) and ELISA kits (detecting PSA in serum and Rac in urine). Material costs of these smart phone based microplate readers were $0.13 \$ 0.19 \$$ (Table S7). Compared with commercial microplate readers, our developed smart phone based microplate readers will be able to make more convenient and cost effective diagnoses. Furthermore, compared with previously reported smart based biosensors, in this system the optical sensor of the smart phone was implemented to measure transmitted light intensities of liquid samples. This enabled more accurate test results. Due to the current optical sensor of the smart phone, it can sense light from $340 \mathrm{~nm}$ to $680 \mathrm{~nm}$. When equipped with different LEDs, smart phone based microplate readers have the potential to readout results of assays with maximum absorption wavelengths between $340 \mathrm{~nm}-680 \mathrm{~nm}$. In future studies, to improve the accuracy of smart phone based microplate readers, more advanced manufactures should be used, such as 3D Print Technology. In addition, a higher resolution smart phone application is essential.

\section{Acknowledgements}


This work was supported by the Guangdong innovative and Entrepreneurial Research Team Program (201301S010 5240297) and the National Science Foundation of China (Grant No.

11204105). For help with English revision, we appreciate the help Charron Cote has offered.

\section{Notes and references}

Barbosa, A.I., Gehlot, P., Sidapra, K., Edwards, A.D., Reis, N.M., 2015. Biosensors and Bioelectronics 70, 5-14.

Bastawrous, A., Armstrong, M.J., 2013. Journal of the royal society of medicine 106(4), 130-142.

Berg, B., Cortazar, B., Tseng, D., Ozkan, H., Feng, S., Wei, Q., Chan, R.Y.-L., Burbano, J., Farooqui, Q., Lewinski, M., 2015. ACS nano 9(8), 7857-7866.

Chadban, S.J., Briganti, E.M., Kerr, P.G., Dunstan, D.W., Welborn, T.A., Zimmet, P.Z., Atkins, R.C., 2003. Journal of the American Society of Nephrology 14(suppl 2), S131S138.

Christodouleas, D.C., Nemiroski, A., Kumar, A.A., Whitesides, G.M., 2015. Analytical chemistry 87(18), 9170-9178.

Coskun, A.F., Ozcan, A., 2014. Current opinion in biotechnology 25, 8-16.

Coskun, A.F., Wong, J., Khodadadi, D., Nagi, R., Tey, A., Ozcan, A., 2013. Lab on a Chip 13(4), 636-640.

Donmez, O., Korkmaz, H.A., Yildiz, N., Ediz, B., 2015. Ren. Fail. 37(5), 784-790.

Drain, P.K., Hyle, E.P., Noubary, F., Freedberg, K.A., Wilson, D., Bishai, W.R., Rodriguez, W., Bassett, I.V., 2014. The Lancet Infectious Diseases 14(3), 239-249.

Fishman, W., Inglis, N.I., Krant, M., 1965. Clinica Chimica Acta 12(3), 298-303.

Fu, Q., Liu, H.L., Wu, Z., Liu, A., Yao, C., Li, X., Xiao, W., Yu, S., Luo, Z., Tang, Y., 2015. Journal of nanobiotechnology 13(1), 81.

Gopinath, S.C., Tang, T.-H., Chen, Y., Citartan, M., Lakshmipriya, T., 2014. Biosensors and Bioelectronics 60, 332-342.

Jia, C.-P., Zhong, X.-Q., Hua, B., Liu, M.-Y., Jing, F.-X., Lou, X.-H., Yao, S.-H., Xiang, J.-Q., Jin, Q.-H., Zhao, J.-L., 2009. Biosensors and Bioelectronics 24(9), 2836-2841.

Kaplan, M.M., 1972. New England Journal of Medicine 286(4), 200-202.

Liang, J., Yao, C., Li, X., Wu, Z., Huang, C., Fu, Q., Lan, C., Cao, D., Tang, Y., 2015. Biosensors and Bioelectronics 69, 128-134.

Ma, H., Li, X., Yan, T., Li, Y., Zhang, Y., Wu, D., Wei, Q., Du, B., 2016. Biosensors and Bioelectronics 79, 379-385.

McNerney, R., Daley, P., 2011. Nature Reviews Microbiology 9(3), 204-213.

Peeling, R., Mabey, D., 2010. Clinical Microbiology and Infection 16(8), 1062-1069.

Pekarthy, J.M., Short, J., Lansing, A.I., Lieberman, I., 1972. Journal of Biological Chemistry 247(6), 1767-1774.

Prati, D., Taioli, E., Zanella, A., Della Torre, E., Butelli, S., Del Vecchio, E., Vianello, L., Zanuso, F., Mozzi, F., Milani, S., 2002. Annals of internal medicine 137(1), 1-10.

Preechaburana, P., Suska, A., Filippini, D., 2014. Trends in biotechnology 32(7), 351355.

Rajendran, V.K., Bakthavathsalam, P., Ali, B.M.J., 2014. Microchimica Acta 181(1516), 1815-1821.

Schaub, S., Rush, D., Wilkins, J., Gibson, I.W., Weiler, T., Sangster, K., Nicolle, L., Karpinski, M., Jeffery, J., Nickerson, P., 2004. Journal of the American Society of Nephrology 15(1), 219-227.

Shu, B., Zhang, C., Xing, D., 2015. Lab on a Chip.

Song, Y., Huang, Y.-Y., Liu, X., Zhang, X., Ferrari, M., Qin, L., 2014. Trends in biotechnology 32(3), 132-139. 
Sorbi, D., Boynton, J., Lindor, K.D., 1999. The American journal of gastroenterology 94(4), 1018-1022.

Tian, B., Bejhed, R.S., Svedlindh, P., Strömberg, M., 2016. Biosensors and Bioelectronics 77, 32-39.

Vashist, S.K., Mudanyali, O., Schneider, E.M., Zengerle, R., Ozcan, A., 2014. Analytical and bioanalytical chemistry 406(14), 3263-3277.

Vashist, S.K., Schneider, E.M., Zengerle, R., von Stetten, F., Luong, J.H., 2015. Biosensors and Bioelectronics 66, 169-176.

Walzik, M.P., Vollmar, V., Lachnit, T., Dietz, H., Haug, S., Bachmann, H., Fath, M., Aschenbrenner, D., Mofrad, S.A., Friedrich, O., 2015. Biosensors and Bioelectronics 64, 639-649.

Wei, Q., Luo, W., Chiang, S., Kappel, T., Mejia, C., Tseng, D., Chan, R.Y.L., Yan, E., Qi, H., Shabbir, F., 2014. ACS nano 8(12), 12725-12733.

Yang, X., Wang, Y., Liu, W., Zhang, Y., Zheng, F., Wang, S., Zhang, D., Wang, J., 2016. Biosensors and Bioelectronics 75, 48-54.

Yu, L., Shi, Z., Fang, C., Zhang, Y., Liu, Y., Li, C., 2015. Biosensors and Bioelectronics 69, 307-315.

Zangheri, M., Cevenini, L., Anfossi, L., Baggiani, C., Simoni, P., Di Nardo, F., Roda, A., 2015. Biosensors and Bioelectronics 64, 63-68.

Zhang, D., Liu, Q., 2016. Biosensors and Bioelectronics 75, 273-284.

Zhu, H., Mavandadi, S., Coskun, A.F., Yaglidere, O., Ozcan, A., 2011. Analytical Chemistry 83(17), 6641-6647.

Zhu, H., Sencan, I., Wong, J., Dimitrov, S., Tseng, D., Nagashima, K., Ozcan, A., 2013. Lab on a Chip 13(7), 1282-1288. 